\title{
Superconvergence of interpolated collocation solutions for weakly singular Volterra integral equations of the second kind
}

\section{Qiumei Huang ${ }^{1} \cdot$ Min $_{W^{2}}{ }^{1}$ (D}

Received: 24 September 2020 / Revised: 5 January 2021 / Accepted: 27 January 2021 /

Published online: 3 March 2021

(c) The Author(s) 2021

\begin{abstract}
In this paper, we discuss the superconvergence of the "interpolated" collocation solutions for weakly singular Volterra integral equations of the second kind. Based on the collocation solution $u_{h}$, two different interpolation postprocessing approximations of higher accuracy: $I_{2 h}^{2 m-1} u_{h}$ based on the collocation points and $I_{2 h}^{m} u_{h}$ based on the least square scheme are constructed, whose convergence order are the same as that of the iterated collocation solution. Such interpolation postprocessing methods are much simpler in computation. We further apply this interpolation postprocessing technique to hybrid collocation solutions and similar results are obtained. Numerical experiments are shown to demonstrate the efficiency of the interpolation postprocessing methods.
\end{abstract}

Keywords Volterra integral equations - Superconvergence - Supercloseness · Interpolation postprocessing $\cdot$ Weakly singular kernels $\cdot$ Collocation $\cdot$ Hybrid collocation

Mathematics Subject Classification 45D05 - 65L70

\section{Introduction}

In this paper, we consider the following weakly singular Volterra integral equation (VIE) of the second kind:

$$
u(t)=g(t)+\int_{0}^{t}(t-s)^{-\alpha} K(t, s) u(s) d s, \quad t \in I:=[0, T]
$$

Communicated by Hui Liang.

Min Wang

w_min@emails.bjut.edu.cn

Qiumei Huang

qmhuang@bjut.edu.cn

1 College of Mathematics, Faculty of Science, Beijing University of Technology, Beijing 100124, China 
with $0<\alpha<1$. $K(t, s), g(t)$ are known functions and $u(t)$ is the function to be determined. Let $D:=\{(t, s): 0 \leq s<t \leq T\}$. We assume that $K(t, s) \in C(D)$, with $K(t, t) \neq 0$ for $t \in I$.

(1) arises in many modeling problems in mathematical physics and chemical reactions, such as stereology, heat conduction, crystal growth, electrochemistry, superfluidity, and the radiation of heat from a semi-infinite solid.

The second kind Volterra integral equations with weakly singular kernels typically have solutions whose derivatives are unbounded at the left endpoint of the interval of integration. Due to this singular behavior, the optimal global and local (super-) convergence results of collocation solutions in piecewise polynomial spaces on uniform meshes will no longer be valid (Brunner 1983). The use of suitable graded meshes (Brunner 1985; Tang 1992) is a possible alternative approach for dealing with this order reduction problem. However, as pointed out in (Brunner 1983; Diogo et al. 1994), the initial stepsize becomes very small in the graded mesh and may cause serious round-off errors when the number of elements $N$ is increased or high-order piecewise polynomials are used. Alternatively, some other numerical methods are proposed to get the optimal convergence of the numerical solutions. See, for example, nonpolynomial spline functions reflecting the singularity on uniform meshes (Brunner 1983), $\beta$-polynomial collocation methods under quasi-graded meshes (Hu 1996, 1997), and variable transformations followed by standard methods (Pedas and Vainikko 2004a, b). The hybrid method first proposed by Cao et al. (2003) uses "looser" graded partitions to avoid round-off errors and nonpolynomial interpolation in the first stepsize to preserve the optimal order of convergence.

Superconvergence is a hot topic in solving various differential equations and integral equations. Based on current convergence results of numerical solutions, suitable postprocessing with relative cheap computation can result in global superconvergence of the new "postprocessed" solutions. There are many postprocessing methods to get the superconvergence of the "postprocessed" solutions of partial differential equations and integral equations. See, for instance, iterated postprocessing (Graham et al. 1985; Sloan 1976, 1990), interpolation postprocessing (Lin and Lin 2006; Lin and Yan 1996), and PPR ( Naga and Zhang 2004; Zhang and Naga 2005), etc. For weakly singular Volterra integral equations, generally, the iterated postprocessing method (including the classical iterative and hybrid iterative methods) is used to accelerate the approximation. See, for example, Diogo 2009; Huang and Xu 2006; Rebelo and Diogo 2010. Interpolation postprocessing was proposed by Lin and his group to accelerate the convergence of finite-element solutions for various partial differential equations, integral equations, and integro-differential equations, and the corresponding works are contained in some papers (such as Lin et al. 1998; Zhang et al. 2000; Huang and Zhang 2010; Huang and Xie 2009) and two monographs (Lin and Lin 2006; Lin and Yan 1996). For weakly singular VIEs, the interpolation postprocessing is used to $\beta$-polynomial collocation solutions to get the superconvergence of the new solutions (Hu 1997). Theoretical analysis and numerical results show that this interpolation postprocessing technique is both simple and of higher accuracy.

In this paper, we apply the interpolation postprocessing technique to the collocation solution under graded mesh and the hybrid collocation solution under "looser" graded mesh to get the same superconvergence as the iterated methods. This interpolation postprocessing is simpler in computation than the iterated postprocessing method. Since the former just needs to interpolate $u_{h}$ at some nearby points, and, however, the latter needs to compute an integral (probably weakly singular) for each $t$.

Here is the outline of the remaining sections. The collocation method, the hybrid collocation method, and the corresponding iterated methods for weakly singular Volterra equations 
are presented in Sect. 2 to make the paper self-contained. In Sect. 3, the supercloseness between the (hybrid) collocation solution and the interpolation of the exact solution $u$ is proved, and the main results about the superconvergence of interpolation postprocessing method, instead of the iterated method, are obtained. Finally, numerical experiments are provided in Sect. 4 to show the efficiency of the interpolation postprocessing method and to compare the computational efficiency of these two postprocessing methods.

\section{Iterated collocation method for weakly singular Volterra integral equations}

In this section, we first introduce the collocation method and the hybrid collocation method for weakly VIEs. The iterated postprocessing is then presented based on these two methods.

\subsection{Collocation method for weakly singular VIEs}

Define the linear Volterra integral operator $\mathcal{V}_{\alpha}$ as:

$$
\left(\mathcal{V}_{\alpha} u\right)(t):=\int_{0}^{t} H_{\alpha}(t, s) u(s) d s, \quad t \in I:=[0, T],
$$

with

$$
H_{\alpha}(t, s):=(t-s)^{-\alpha} K(t, s), \quad 0<\alpha<1 .
$$

Then, the corresponding operator form of the linear Volterra integral equation (1) is as follows:

$$
u(t)=g(t)+\left(\mathcal{V}_{\alpha} u\right)(t), \quad t \in I .
$$

The existence and uniqueness of the solution for (1) (or (4)) are given in the following Theorem (see Brunner (2004) for details).

Theorem 1 Assume that $K \in C(D)$ and $0<\alpha<1$. Then, for any $g \in C(I)$ the linear, weakly singular Volterra integral equation (1) possesses a unique solution $u \in C(I)$. If $g \in C^{m}(I)$ and $K \in C^{m}(D)$ with $K(t, t) \neq 0$ on $I$, then:

$$
u \in C^{m}(0, T] \cap C(I), \text { with }\left|u^{\prime}(t)\right| \leq C_{\alpha} t^{-\alpha}, \text { for } t \in(0, T] .
$$

Typically, VIEs with weakly singular kernels behave like $t^{-\alpha}(0<\alpha<1)$ have solutions whose derivatives are unbounded at the left endpoint of the interval of integration. Due to this singular behavior, the optimal global and local (super-) convergence results for collocation solutions in piecewise polynomial spaces on uniform meshes will no longer be valid. The use of appropriately graded meshes is one of the possible alternative approaches for dealing with this order reduction.

We recall that for an interval $I:=[0, T]$, a graded mesh with grading exponent $r>1$ is defined by:

$$
I_{h}:=\left\{t_{n}=t_{n}^{(N)}:=(n / N)^{r} T, n=0,1, \ldots, N\right\} .
$$

The sequence $\left\{h_{n}=t_{n}-t_{n-1}(n=1,2, \ldots, N)\right\}$ is strictly increasing.

The piecewise polynomial space of degree $\leq m-1$ is defined as:

$$
S_{m-1}^{(-1)}\left(I_{h}\right):=\left\{v \in L^{2}(I):\left.v\right|_{I_{n}} \in \mathcal{P}_{m-1}(1 \leq n \leq N)\right\},
$$


where $\mathcal{P}_{m-1}$ is the space of all polynomials of degree $\leq m-1$.

The desired collocation solution $u_{h}$ for the weakly singular VIE (4) is defined by:

$$
u_{h}(t)=g(t)+\left(\mathcal{V}_{\alpha} u_{h}\right)(t), \quad t \in X_{h},
$$

where the set of collocation points:

$$
\begin{aligned}
X_{h}:= & \left\{t_{n i}=t_{n-1}+c_{i} h_{n}: 0<c_{1}<\ldots<c_{i}<\ldots<c_{m} \leq 1,\right. \\
& n=1,2, \ldots, N ; i=1,2, \ldots, m\} .
\end{aligned}
$$

is determined by the given mesh $I_{h}$ and the (distinct) collocation parameters $\left\{c_{i}\right\}$.

Define the corresponding interpolatory projection operator $P_{h}: C[0, T] \rightarrow S_{m-1}^{(-1)}\left(I_{h}\right)$ satisfying that for $x \in C[0, T]$ :

$$
P_{h}\left(x\left(t_{n, i}\right)\right)=x\left(t_{n, i}\right)(n=1,2, \ldots, N ; i=1, \ldots, m) .
$$

It is easy to verify that $P_{h}$ is bounded. The collocation equation (6) has the following operator form:

$$
u_{h}=P_{h} g+P_{h}\left(\mathcal{V}_{\alpha} u_{h}\right)
$$

Theorem 2 (Brunner 2004) Assume:

1. The given functions in the Volterra integral equation (1) satisfy $K \in C^{m}(D)$ and $g \in$ $C^{m}(I)$.

2. $u_{h} \in S_{m-1}^{(-1)}\left(I_{h}\right)$ is the (unique) collocation solution to (1) defined by (6), with $h:=$ $T / N \in(0, \bar{h})$ and corresponding to the collocation points $X_{h}$, where $\bar{h}=\bar{h}(\alpha)>0$.

3. The grading exponent $r=r(\alpha) \geq 1$ determining the mesh $I_{h}$ is given by:

$$
r(\alpha)=\frac{\mu}{1-\alpha}, \quad \mu \geq 1-\alpha .
$$

Then, we have:

$$
\left\|u-u_{h}\right\|_{\infty}:=\sup _{t \in I}\left|u(t)-u_{h}(t)\right| \leq C(r) \begin{cases}h^{\mu}, & \text { if } 1-\alpha \leq \mu<m, \\ h^{m}, & \text { if } \mu \geq m,\end{cases}
$$

holds for any set $X_{h}$ of collocation points with $0 \leq c_{1}<\cdots<c_{m} \leq 1$. The constant $C(r)$ depends on $\left\{c_{i}\right\}$ and on the grading exponent $r=r(\alpha)$, but not on $h$.

\subsection{Hybrid collocation method for weakly singular VIEs}

Although the graded mesh can get the desired convergence, its initial stepsize becomes very small and may cause round-off errors for $\alpha \rightarrow 1$ when $N$ is increased or high-order piecewise polynomials are used. A "looser" graded mesh combining nonpolynomials interpolation in the initial stepsize called the hybrid method (Cao et al. 2003) was proposed to optimize the grid while maintaining the same accuracy.

In this subsection, we describe the "looser" graded partition of $I$. Specifically, for a real number $r \geq 1$, we let $i_{0}$ be an integer, such that:

$$
\left\lceil\left(\frac{N}{i_{0}}\right)^{r}\right\rceil=N
$$

where $\lceil a\rceil$ denotes the smallest integer greater than or equal to $a$.

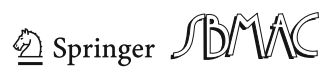


It is easy to verify that such an integer $i_{0}$ exists and satisfies:

$$
N^{1-1 / r} \leq i_{0}<N(N-1)^{-1 / r}
$$

or the equivalent estimate:

$$
N^{1-1 / r} \leq i_{0}<N^{1-1 / r}+1 .
$$

Set $N^{\prime}:=N-i_{0}+1$. The partition on $I$ is given by:

$$
t_{0}=0, t_{i}=\left(\frac{i_{0}+i-1}{N}\right)^{r} T, i=1,2, \ldots, N^{\prime} .
$$

Note that $t_{N^{\prime}}=T$ and the stepsize of the first subinterval for this partition is larger than that for the graded mesh and reduces the possibility of round errors.

However, this "looser" partition cannot capture the singularity of the exact solution as well as the graded mesh due to the "bigger" stepsize of the initial subinterval. To preserve the singularity properties of the solution, the nonpolynomial interpolation reflecting the singularities of the exact solution is used in the first subinterval.

For $0<\alpha<1$ and a positive integer, we introduce an index set:

$$
W_{\alpha, m}=\left\{i+j \alpha: i+j \alpha<m, i, j \in N^{*}\right\},
$$

where $N^{*}$ denotes the set of the nonnegative integers and $l$ is the cardinality of the dimension of the space $W_{\alpha, m}\left(l:=\operatorname{dim} W_{\alpha, m}\right)$.

The nonpolynomial space $V_{m}$ of degree $<m$ is defined by:

$$
V_{m}:=\operatorname{span}\left\{t^{\nu_{j}}: j=0,1, \ldots, l-1, v_{j} \in W_{\alpha, m}\right\} .
$$

The hybrid space $S_{m}^{(-1)}\left(I_{h}\right)$ of degree $<m$ is defined by:

$$
S_{m}^{(-1)}\left(I_{h}\right):=\left\{v \in L^{2}(I):\left.v\right|_{I_{1}} \in V_{m},\left.v\right|_{I_{i}} \in \mathcal{P}_{m-1}, i=2,3, \cdots, N^{\prime}\right\} .
$$

Let $0 \leq c_{1}<c_{2}<\ldots<c_{l} \leq 1$, and we choose $l$ collocation points $t_{1 j}:=t_{0}+c_{j} h_{1}(j=$ $1,2, \ldots, l)$ in $I_{1}=\left[t_{0}, t_{1}\right]$ and $t_{i j}=t_{i-1}+c_{j} h_{i}(j=1,2, \ldots, m)$ in $I_{i}=\left[t_{i-1}, t_{i}\right](i=$ $\left.2, \ldots, N^{\prime}\right)$, and denoted by:

$$
\bar{X}_{h}=\left\{\begin{array}{l}
t_{1 j}:=t_{0}+c_{j} h_{1}(j=1,2, \ldots, l), t_{1 j} \in I_{1} \\
t_{i j}=t_{i-1}+c_{j} h_{i}(j=1,2, \ldots, m), t_{i j} \in I_{i}, i=2, \ldots, N^{\prime} .
\end{array}\right.
$$

For simplicity of the notations, we introduce the notation:

$$
m_{n}:= \begin{cases}l, & n=1 \\ m, & n=2,3, \ldots, N^{\prime} .\end{cases}
$$

The hybrid collocation method for (1) [or the relevant operator form (4)] is to seek $u_{h} \in$ $S_{m}^{(-1)}\left(I_{h}\right)$, such that:

$$
u_{h}\left(t_{n j}\right)=g\left(t_{n j}\right)+\left(\mathcal{V}_{\alpha} u_{h}\right)\left(t_{n j}\right), n=1,2, \ldots, N^{\prime}, j=1,2, \ldots, m_{n} .
$$

For $f \in C(I)$, we define the relevant hybrid interpolation operator $Q_{h}: C(I) \rightarrow S_{m}^{(-1)}\left(I_{h}\right)$ by:

$$
\left(Q_{h} f\right)\left(t_{n i}\right)=f\left(t_{n i}\right), \quad n=1,2, \cdots, N^{\prime}, j=1,2, \cdots, m_{n} .
$$

We know from the definition of $Q_{h}$ that the singularity preserving (nonpolynomial) interpolation is used in the first subinterval and the standard piecewise polynomial interpolation in the rest of subintervals. We refer (Cao et al. 2003) for more details of $Q_{h}$. 
Then, the operator form of the hybrid collocation method for weakly singular VIE (4) or (1) is to seek $u_{h} \in S_{m}^{(-1)}\left(I_{h}\right)$, such that:

$$
u_{h}=Q_{h} g+Q_{h}\left(\mathcal{V}_{\alpha} u_{h}\right)
$$

where $Q_{h}$ is defined by (15) under the mesh defined by (11).

The following lemma shows the convergence of the hybrid collocation solution.

Lemma 1 (Cao et al. 2003) Let $u$ be the exact solution of (1) and $N$ be a positive integer. Let $Q_{h}$ be the hybrid interpolation operator defined by (15) associated with the graded partition (11). Suppose the forcing function $g$ in (1) has the form $g(t)=\sum_{j+i \alpha<m} g_{i j} t^{j+i \alpha}+g_{m}(t)$.

Then, for sufficiently large $N$, (16) has a unique solution $u_{h}$ and there exists a positive constant $C$ independent of $N$, such that:

$$
\left\|u-u_{h}\right\|_{\infty} \leq C N^{-m}
$$

\subsection{Iterated postprocessing for (hybrid) collocation solutions}

The iterated collocation solution $u_{h}^{i t}$ corresponding to the (hybrid) collocation solution $u_{h}$ is then defined by:

$$
u_{h}^{i t}(t):=g(t)+\left(\mathcal{V}_{\alpha} u_{h}\right)(t), \quad t \in I .
$$

It trivially satisfies:

$$
u_{h}^{i t}(t)=u_{h}(t), \quad \forall t \in X_{h}\left(\bar{X}_{h}\right) .
$$

That is, $P_{h} u_{h}^{i t}=u_{h}$ for the traditional collocation method and $Q_{h} u_{h}^{i t}=u_{h}$ for the hybrid collocation method.

It is easy to prove that the iterated collocation solution $u_{h}^{i t}$ have the following operator form:

$$
u_{h}^{i t}=g+\mathcal{V}_{\alpha}\left(R_{h} u_{h}^{i t}\right)
$$

where the operator $R_{h}:=P_{h}$ for the traditional collocation method and $R_{h}:=Q_{h}$ for the hybrid collocation method.

Next, we give the superconvergence results of iterated collocation solutions and iterated hybrid collocation solutions.

Theorem 3 (Brunner 2004) Assume:

1. $g \in C^{m+1}(I), K \in C^{m+1}(D)$, with $K(t, t) \neq 0$ on $I$, and $0<\alpha<1$;

2. $u_{h} \in S_{m-1}^{(-1)}\left(I_{h}\right)$ is the (unique) collocation solution to (1), with corresponding iterated collocation solution $u_{h}^{i t}$;

3. the collocation parameters satisfy $J_{0}:=\int_{0}^{1} \prod_{i=1}^{m}\left(s-c_{i}\right) d s=0$;

4. $I_{h}$ is the graded mesh (5) with grading exponent $r=r(\alpha) \geq 1$ and $h:=T / N$.

Then:

$$
\left\|u-u_{h}^{i t}\right\|_{\infty} \leq C(r) \begin{cases}h^{2(1-\alpha)}, & \text { if } r=1, \\ h^{m+1-\alpha}, & \text { if } r \geq \frac{m}{1-\alpha} .\end{cases}
$$


Similarly, the hybrid collocation parameters is supposed to satisfy:

$$
J_{0}:=\int_{0}^{1} s^{j} \prod_{i=1}^{m}\left(s-c_{i}\right) d s=0, \quad\left(j=0, \ldots, r_{0}\right),
$$

where $r_{0}$ is a nonnegative integer less than $m$. When $r_{0}=1$, the following result for the iterated hybrid collocation method is obtained.

Theorem 4 (Huang and $\mathrm{Xu}$ 2006) Suppose that $K \in C^{m+2}(D)$ and $g \in C^{m+2}(I)$. Let u be the exact solution of (1) and $u_{h} \in S_{m}^{(-1)}\left(I_{h}\right)$ is the (unique) hybrid collocation solution to (1), with corresponding iterated hybrid collocation solution $u_{h}^{i t}$. Then, there exists a positive constant $\mathrm{C}$ and a positive integer $N_{0}$, such that for all $N>N_{0}$ :

$$
\left\|u-u_{h}^{i t}\right\|_{\infty} \leq C N^{-m-1+\alpha} .
$$

If $r_{0}=0$ in the orthogonal condition (21), we can get the similar result of iterated hybrid collocation solutions under the same regularity restrictions of $K, g$ as Theorem 3:

Corollary 1 Assume that $g \in C^{m+1}(I), K \in C^{m+1}(D)$, with $K(t, t) \neq 0$, the hybrid collocation parameters satisfy $\int_{0}^{1} \prod_{i=1}^{m}\left(s-c_{i}\right) d s=0$. Then, for the iterated hybrid collocation solution, there holds:

$$
\left\|u-u_{h}^{i t}\right\|_{\infty} \leq C \begin{cases}N^{-(m+1-\alpha)}, & \text { if } \alpha \geq \frac{1}{m+1}, \\ N^{-\left(m+1-\frac{1-\alpha}{m}\right)}, & \text { if } \quad \alpha<\frac{1}{m+1}\end{cases}
$$

In fact, when $r_{0}=0$, (21) simplifies to $\int_{0}^{1} \prod_{i=1}^{m}\left(s-c_{i}\right) d s=0$.

The kernel $H_{\alpha}(t, s)$ satisfies the following condition on $D=\{(t, s): 0 \leq s \leq t \leq T\}$ :

$$
\left|\frac{\partial^{i}}{\partial s^{i}} H_{\alpha}(t, s)\right| \leq C(t-s)^{-\alpha-i}, \quad i=0,1, \ldots, m .
$$

See (3.2) of Huang and $\mathrm{Xu}$ (2006) for details, where $l=-1, \beta=0$. The conclusion is valid by Lemma 3.2 and (3.23) of Huang and $\mathrm{Xu}$ 2006. We leave the details to the reader.

Remark 1 We see from Lemma 1 and Theorem 4 that the graded parameter $r$ of the hybrid collocation method is not as sensitive to the values of $m, \alpha$ as the traditional collocation method.

\section{Global superconvergence of interpolation postprocessing}

In this section, some results on supercloseness between the (hybrid) collocation solution $u_{h}$ and the interpolant defined in (8) [(15) for hybrid collocation] of the exact solution $u$ are given, and then, we use two postprocessing methods based on the supercloseness to accelerate the (hybrid) collocation solutions.

\subsection{Supercloseness analysis between $u_{h}$ and $P_{h} u\left(Q_{h} u\right)$}

We begin by citing from (Lin and Lin 2006; Lin and Yan 1996) the following definition of "supercloseness". 
Definition 1 If the error between the numerical solution and some interpolant of the exact solution is much smaller than that between the numerical solution and the exact solution; that is, if:

$$
\left\|P_{h} u-u_{h}\right\|_{\infty} \ll\left\|u-u_{h}\right\|_{\infty},
$$

then this phenomenon is called "supercloseness".

We then introduce the following Hölder space:

$$
C^{(0, \beta)}[a, b]=\left\{g \in C[a, b] \mid d_{\beta}(g) \equiv \sup _{a \leq t, \tau \leq b} \frac{|g(t)-g(\tau)|}{|t-\tau|^{\beta}}<\infty\right\},
$$

where $0<\beta<1$, and if $u \in C^{(0, \beta)}[a, b]$, then $u$ is called $\beta-H \ddot{o l d e r}$ continuous.

Lemma 2 Atkinson 1997 For $N, m>0$ and grading exponent $r \geq 1, P_{h}$ is the interpolation projection operator defined by (8) in Sect. 2. For $0<\alpha<1$, assume $u \in C^{(0,1-\alpha)}[0,1] \cap$ $C^{m}(0,1]$, with:

$$
\left|u^{m}(t)\right| \leq c_{\alpha, m}(u) t^{1-\alpha-m}, \quad 0<t \leq 1,
$$

where $C^{(0,1-\alpha)}[0,1]$ is the Hölder space,

Then, for $r \geq \frac{m}{1-\alpha}$, we have:

$$
\left\|u-P_{h} u\right\|_{\infty} \leq \frac{c}{N^{m}}
$$

with $\mathrm{c}$ a constant independent $N$.

Theorem 5 Under the conditions stated in Theorem 3, the following global supercloseness result holds:

$$
\left\|u_{h}-P_{h} u\right\|_{\infty} \leq C(r) \begin{cases}h^{2(1-\alpha)}, & \text { if } r=1, \\ h^{m+1-\alpha}, & \text { if } \quad r \geq \frac{m}{1-\alpha} .\end{cases}
$$

Proof We know from (4) and (9) that:

$$
\begin{aligned}
u_{h}-P_{h} u & =P_{h} g+P_{h}\left(\mathcal{V}_{\alpha} u_{h}\right)-\left(P_{h} g+P_{h}\left(\mathcal{V}_{\alpha} u\right)\right) \\
& =P_{h} \mathcal{V}_{\alpha}\left(u_{h}-u\right)=P_{h} \mathcal{V}_{\alpha}\left(u_{h}-P_{h} u+P_{h} u-u\right) ;
\end{aligned}
$$

then:

$$
u_{h}-P_{h} u=\left(I-P_{h} \mathcal{V}_{\alpha}\right)^{-1} P_{h} \mathcal{V}_{\alpha}\left(P_{h} u-u\right)
$$

For the first subinterval, i.e., for $n=1$, we combine Lemma 2 and Theorem 1 that:

$$
\begin{aligned}
\left\|\mathcal{V}_{\alpha}\left(P_{h} u-u\right)\right\|_{I_{1}, \infty} & =\max _{t \in\left[0, t_{1}\right]}\left|\int_{0}^{t}(t-s)^{-\alpha} K(t, s)\left(P_{h} u-u\right)(s) d s\right| \\
& \leq \max _{t, s \in D}|K(t, s)|\left\|P_{h} u-u\right\|_{I_{1}, \infty} \max _{t \in\left[0, t_{1}\right]}\left|\int_{0}^{t}(t-s)^{-\alpha} d s\right| \\
& \leq C\left\|P_{h} u-u\right\|_{I_{1}, \infty} \max _{t \in\left[0, t_{1}\right]}\left|\int_{0}^{t}(t-s)^{-\alpha} d s\right| \\
& =C \max _{\left.t \in\left[0, t_{1}\right]\right)}\left|\frac{t^{1-\alpha}}{1-\alpha}\right|\left\|P_{h} u-u\right\|_{I_{1}, \infty} \leq C \frac{h_{1}^{1-\alpha}}{1-\alpha} h_{1}^{1-\alpha}
\end{aligned}
$$




$$
\begin{aligned}
& \leq C h^{1-\alpha}\left(\frac{1}{N}\right)^{r(1-\alpha)} \leq C h^{(1+r)(1-\alpha)} \\
& \leq\left\{\begin{array}{l}
h^{2(1-\alpha)}, \text { if } r=1, \\
h^{m+1-\alpha}, \text { if } r \geq \frac{m}{1-\alpha} .
\end{array}\right.
\end{aligned}
$$

For $n \geq 2$, we write $t \in I_{n}$ as $t=t_{n-1}+v h_{n}$ with $v \in[0,1]$. Then:

$$
\begin{aligned}
\mathcal{V}_{\alpha}\left(P_{h} u-u\right)(t) \\
=\int_{0}^{t_{n-1}}(t-s)^{-\alpha} K(t, s)\left(P_{h} u-u\right)(s) d s \\
\quad+h_{n}^{1-\alpha} \int_{0}^{v}(v-s)^{-\alpha} K\left(t_{n-1}+v h_{n}, t_{n-1}+s h_{n}\right)\left(P_{h} u-u\right)\left(t_{n-1}+s h_{n}\right) d s .
\end{aligned}
$$

For the second term of the right-hand side of (25), by Lemma 2, we obtain:

$$
\begin{aligned}
& h_{n}^{1-\alpha}\left|\int_{0}^{v}(v-s)^{-\alpha} K\left(t_{n-1}+v h_{n}, t_{n-1}+s h_{n}\right)\left(P_{h} u-u\right)\left(t_{n-1}+s h_{n}\right) d s\right| \\
\leq & h_{n}^{1-\alpha} \max _{t, s \in D}|K(t, s)|\left\|P_{h} u-u\right\|_{\infty}\left|\int_{0}^{v}(v-s)^{-\alpha} d s\right| \\
\leq & C h_{n}^{1-\alpha}\left\|P_{h} u-u\right\|_{\infty}\left|\int_{0}^{v}(v-s)^{-\alpha} d s\right| \\
= & C h_{n}^{1-\alpha}\left\|P_{h} u-u\right\|_{\infty} \frac{v^{1-\alpha}}{1-\alpha} \\
\leq & C h^{1-\alpha}\left\|P_{h} u-u\right\|_{\infty} \leq C h^{1-\alpha} \cdot h^{m} \\
\leq & C h^{m+1-\alpha} .
\end{aligned}
$$

The first term of the right-hand side of (25) can be written as:

$$
\begin{aligned}
\mid & \int_{0}^{t_{n-1}}(t-s)^{-\alpha} K(t, s)\left(P_{h} u-u\right)(s) d s \mid \\
= & \left|\sum_{l=1}^{n-1} \int_{t_{l}-1}^{t_{l}}(t-s)^{-\alpha} K(t, s)\left(P_{h} u-u\right)(s) d s\right| \\
= & \left|\sum_{l=1}^{n-1} h_{l}^{1-\alpha} \int_{0}^{1}\left(\frac{t_{n-1}+v h_{n}-t_{l-1}}{h_{l}}-s\right)^{-\alpha} K\left(t_{n-1}+v h_{n}, t_{l-1}+s h_{l}\right)\left(P_{h} u-u\right)\left(t_{l-1}+s h_{l}\right) d s\right| \\
= & \mid \sum_{l=1}^{n-1} h_{l}^{1-\alpha}\left(\sum_{j=1}^{m} \omega_{j}\left(\frac{t_{n-1}+v h_{n}-t_{l-1}}{h_{l}}-c_{j}\right)^{-\alpha} K\left(t_{n-1}+v h_{n}, t_{l-1}+c_{j} h_{l}\right)\left(P_{h} u-u\right)\left(t_{l-1}+c_{j} h_{l}\right)\right. \\
& \left.+E_{l}(t)\right) \mid,
\end{aligned}
$$

where $\omega_{j}$ is the weight function, and we write the integral into the numerical quadrature with the reminder $E_{l}(t),\left|E_{l}(t)\right| \leq C h^{d}(d \geq m+1)$.

From the definition of $P_{h}$, we know that:

$$
\left(P_{h} u-u\right)\left(t_{l-1}+c_{j} h_{l}\right)=0 .
$$

Therefore:

$$
\left|\int_{0}^{t_{n-1}}(t-s)^{-\alpha} K(t, s)\left(P_{h} u-u\right)(s) d s\right|=\left|\sum_{l=1}^{n-1} h_{l}^{1-\alpha} E_{l}(t)\right|
$$




$$
\begin{aligned}
& \leq C h^{1-\alpha} \sum_{l=1}^{n-1} h_{l}^{m+1} \leq C h^{m+1-\alpha} \sum_{l=1}^{n-1} h_{l} \\
& \leq C h^{m+1-\alpha},
\end{aligned}
$$

where it is easy to verify that $h_{l} \leq C h$.

Hence, for $n \geq 1$, we have:

$$
\begin{aligned}
\left\|u_{h}-P_{h} u\right\|_{\infty} & =\left\|\left(I-P_{h} \mathcal{V}_{\alpha}\right)^{-1} P_{h} \mathcal{V}_{\alpha}\left(P_{h} u-u\right)\right\|_{\infty} \\
& \leq C h^{m+1-\alpha}
\end{aligned}
$$

Theorem 6 Under the conditions stated in Corollary 1, we have the following supercloseness between the hybrid collocation $u_{h}$ and the hybrid interpolation $Q_{h} u$ of the exact solution $u$ :

$$
\left\|u_{h}-Q_{h} u\right\|_{\infty} \leq C\left\{\begin{array}{ll}
N^{-(m+1-\alpha)}, & \text { if } \alpha \geq \frac{1}{m+1}, \\
N^{-\left(m+1-\frac{1-\alpha}{m}\right)}, & \text { if } \alpha<\frac{1}{m+1}
\end{array} .\right.
$$

The proof is similar with the iterated hybrid collocation method in Huang and $\mathrm{Xu}$ (2006) (Theorem 3.4), since $u_{h}-Q_{h} u=\left(I-Q_{h} \mathcal{V}_{\alpha}\right)^{-1} Q_{h} \mathcal{V}_{\alpha}\left(Q_{h} u-u\right)$ and $u-u_{h}^{i t}=(I-$ $\left.\mathcal{V}_{\alpha} Q_{h}\right)^{-1} \mathcal{V}_{\alpha}\left(u-Q_{h} u\right)$. We omit the details and leave the proof to the reader.

Remark 2 The conclusions in Theorem 5 and Theorem 6 can also be obtained by the results of the iterated collocation and iterated hybrid collocation solutions. In fact, from the relationship between $u_{h}$ and $u_{h}^{i t}$, we know that:

$$
u_{h}-P_{h} u=P_{h} u_{h}^{i t}-P_{h} u=P_{h}\left(u_{h}^{i t}-u\right) .
$$

Then:

$$
\left\|u_{h}-P_{h} u\right\|_{\infty}=\left\|P_{h}\right\|_{\infty}\left\|u_{h}^{i t}-u\right\|_{\infty} \leq C\left\|u_{h}^{i t}-u\right\|_{\infty} .
$$

(20) leads to the supercloseness results. We replace $P_{h}$ by $Q_{h}$ and the relevant supercloseness results of the hybrid collocation method can be obtained in the same way.

\subsection{Global superconvergence of interpolation collocation solutions}

Based on the supercloseness between $u_{h}$ and $P_{h} u$, we can obtain the global superconvergence of the new "interpolated collocation solution" by applying the interpolation postprocessing technique to the collocation solution instead of the iterated collocation method.

We assume that $I_{h}$ is gained from $I_{2 h}$ with mesh size $2 h$ by subdividing each element of $I_{2 h}$ into two equal elements, so that the number of elements $N$ for $I_{h}$ is even. Then, we define a higher order interpolation operator $I_{2 h}^{2 m-1}$ of degree $(2 m-1)$ associated with $I_{2 h}$ satisfying the following conditions:

$$
\left.I_{2 h}^{2 m-1} u\right|_{I_{i} \cup I_{i+1}} \in \mathcal{P}_{2 m-1} \quad(i=1,3, \cdots, N-1),
$$

and

$$
\begin{aligned}
& I_{2 h}^{2 m-1} u\left(t_{n i}\right)=u\left(t_{n i}\right), \\
& I_{2 h}^{2 m-1} u\left(t_{(n+1) i}\right)=u\left(t_{(n+1) i}\right), \quad(n=1,3, \ldots, N-1 ; i=1,2, \ldots, m) .
\end{aligned}
$$


It is easy to check that:

$$
\begin{aligned}
\left\|v-I_{2 h}^{2 m-1} v\right\|_{\infty} & \leq C h^{m+1}\|v\|_{m+1, \infty}, \quad \forall v \in W^{m+1, \infty}(I) ; \\
I_{2 h}^{2 m-1} P_{h} & =I_{2 h}^{2 m-1} ; \\
\left\|I_{2 h}^{2 m-1} v\right\|_{\infty} & \leq C\|v\|_{\infty}, \quad \forall v \in S_{m-1}^{(-1)}\left(I_{h}\right) .
\end{aligned}
$$

Thus, we can get the global superconvergence of the new "interpolated collocation solution" by the interpolation postprocessing method as follows.

Theorem 7 Under the conditions stated in Theorem 3 and assume that $u \in W^{m+1, \infty}(I)$, the following global superconvergence result holds:

$$
\left\|I_{2 h}^{2 m-1} u_{h}-u\right\|_{\infty} \leq C(r)\left\{\begin{array}{ll}
h^{2(1-\alpha)}, & \text { if } r=1, \\
h^{m+1-\alpha}, & \text { if } r \geq \frac{m}{1-\alpha}
\end{array} .\right.
$$

Proof It follows from (30), the interpolation error estimates, and (23) that:

$$
\begin{aligned}
\left\|I_{2 h}^{2 m-1} u_{h}-u\right\|_{\infty} & \leq\left\|I_{2 h}^{2 m-1} u_{h}-I_{2 h}^{2 m-1} P_{h} u\right\|_{\infty}+\left\|I_{2 h}^{2 m-1} P_{h} u-u\right\|_{\infty} \\
& \leq C\left\|u_{h}-P_{h} u\right\|_{\infty}+\left\|I_{2 h}^{2 m-1} u-u\right\|_{\infty} \\
& \leq C\left\|u_{h}-P_{h} u\right\|_{\infty}+C h^{m+1} \\
& \leq C(r)\left\{\begin{array}{l}
h^{2(1-\alpha)}, \text { if } r=1, \\
h^{m+1-\alpha}, \text { if } r \geq \frac{m}{1-\alpha} .
\end{array}\right.
\end{aligned}
$$

We observe that the degree $(2 m-1)$ is not the unique choice for the interpolation postprocessing operator. In fact, the superconvergence result (31) is valid for any interpolation operator $I_{2 h}^{p}(p \geq m)$ that satisfies (28)-(30).

In contrast to (26)-(27), the operator $I_{2 h}^{p}$ can also be constructed by the least-squares method (Naga and Zhang 2004; Zhang and Naga 2005).

Polynomial Preserving Recovery (PPR). In this scheme, the higher order interpolation operator $I_{2 h}^{m}$ of degree $m$ associated with $I_{2 h}$ is:

$$
\left.I_{2 h}^{m} u\right|_{I_{n} \cup I_{n+1}} \in \mathcal{P}_{m} \quad(n=1,3, \ldots, N-1) .
$$

In each bigger subinterval $I_{n} \cup I_{n+1}, I_{2 h}^{m} u$ is the solution of the least-squares problem:

$$
\sum_{i=1}^{2 m}\left|u\left(t_{i}^{*}\right)-I_{2 h}^{m} u\left(t_{i}^{*}\right)\right|^{2}=\min _{v \in \mathcal{P}_{m}\left(I_{n} \cup I_{n+1}\right)} \sum_{i=1}^{2 m}\left|u\left(t_{i}^{*}\right)-v\left(t_{i}^{*}\right)\right|^{2},
$$

where $t_{i}^{*}(i=1, \cdots, 2 m)$ are the $2 m$ collocation points in $I_{n}$ and $I_{n+1}(n=1,3, \ldots, N-1)$. It is readily verified that this type of interpolation also satisfies the conditions (28)-(30).

Theorem 8 Let the conditions stated in Theorem 3 hold and assume that $u \in W^{m+1, \infty}(I)$. The interpolation operator $I_{2 h}^{m}$ is defined by (32)-(33), and then, the following global superconvergence estimate holds:

$$
\left\|I_{2 h}^{m} u_{h}-u\right\|_{\infty} \leq C(r)\left\{\begin{array}{l}
h^{2(1-\alpha)}, \text { if } r=1, \\
h^{m+1-\alpha}, \text { if } r \geq \frac{m}{1-\alpha} .
\end{array}\right.
$$

The proof is similar with Theorem 7 . We omit here and leave it to the reader. 


\subsection{Global superconvergence for interpolated hybrid collocation solutions}

Based on the supercloseness between $u_{h}$ and $Q_{h} u$, we can obtain the global superconvergence of the new "interpolated hybrid collocation solution" by applying the interpolation postprocessing to the hybrid collocation solution.

Similar with Sect. 3.2, we assume the number of elements $N$ for $I_{h}$ is an even number. We define the higher order interpolation operator $\hat{I}_{2 h}:=\tilde{I}_{2 h}^{m+1} \cup I_{2 h}^{2 m-1}$, where $\tilde{I}_{2 h}^{m+1}$ denotes the nonpolynomial interpolation of degree $<m+1$ in $I_{1} \cup I_{2}$ and $I_{2 h}^{2 m-1}$ denotes the normal higher order polynomial interpolation of degree $(2 m-1)$ associated with $\left[t_{2}, T\right]$ satisfying the following conditions:

$$
\begin{aligned}
\left.\tilde{I}_{2 h}^{m+1} u\right|_{I_{1} \cup I_{2}} & \in V_{m+1}, \\
\left.I_{2 h}^{2 m-1} u\right|_{I_{i} \cup I_{i+1}} & \in P_{2 m-1} \quad(i=3,5, \ldots, N-1),
\end{aligned}
$$

and

$$
\begin{aligned}
& \tilde{I}_{2 h}^{m+1} u\left(t_{1 i}\right)=u\left(t_{1 i}\right), \quad(i=1,2 \ldots, l) . \\
& \tilde{I}_{2 h}^{m+1} u\left(t_{2 i}\right)=u\left(t_{2 i}\right), \quad(i=1,2 \ldots, m) . \\
& I_{2 h}^{2 m-1} u\left(t_{n i}\right)=u\left(t_{n i}\right), \\
& I_{2 h}^{2 m-1} u\left(t_{(n+1) i}\right)=u\left(t_{(n+1) i}\right), \quad(n=3,5, \ldots, N-1 ; i=1,2 \ldots, m) .
\end{aligned}
$$

where $V_{m+1}$ is the nonpolynomial space of degree $<m+1$ defined in the hybrid collocation method.

We note that when we get the "better" approximation based on the hybrid collocation solution, the first "bigger" subinterval $\tilde{I}_{1}=I_{1} \cup I_{2}$ need to be paid attention. For the "bigger" new subinterval $\tilde{I}_{1}$, we compute the interpolated hybrid collocation solution using the singularity preserving (nonpolynomial) interpolation rather than an easy addition of nonpolynomial interpolation and piecewise polynomial interpolation. We use the $l+m$ collocation points to get the nonpolynomial interpolation $\tilde{I}_{2 h}^{m+1} u$ of degree $<m+1$ in $\tilde{I}_{1}$. Let $M:=\operatorname{dim} V_{m+1}$. Normally, $M \neq l+m$. The least-squares method is usually used to get $\tilde{I}_{2 h}^{m+1} u$ by the interpolation conditions (35) and (36).

It is easy to verify that $\hat{I}_{2 h}$ of (35)-(38) satisfies (28)-(30), with $P_{h}$ replaced by $Q_{h}$.

Thus, we can get the global superconvergence result of the new "interpolated hybrid collocation solution" by applying the interpolation postprocessing to the "hybrid collocation solution".

Theorem 9 Under the conditions stated in Theorem 3, the following global superconvergence result holds:

$$
\left\|\hat{I}_{2 h} u_{h}-u\right\|_{\infty} \leq C N^{-m-1+\alpha}
$$

The proof is similar with the proof of Theorem 7. We omit here and leave it to the reader.

Similarly, the postprocessing operator $\tilde{I}_{2 h}$ is not the unique choice and the superconvergence result (39) is valid for any interpolation operator $I_{2 h}^{p}(p \geq m)$ that satisfies (35)-(38). 


\subsection{Interpolation postprocessing VS iteration postprocessing}

In this subsection, we compare the computational complexities of the two kinds of postprocessings. For simplicity, we only take the interpolation postprocessing $I_{2 h}^{2 m-1} u_{h}$ and the iterated postprocessing $u_{h}^{i t}$ on the collocation solution as an example.

Interpolation postprocessing solution $I_{2 h}^{2 m-1} u_{h}(t)$.

We get the "better" approximation $I_{2 h}^{2 m-1} u_{h}$ of the exact solution $u$ by combining the adjacent subinterval and constructing higher order polynomials in each "bigger" subintervals $\tilde{I}_{\frac{n+1}{2}}:=I_{n} \cup I_{n+1}(n=1,3, \cdots, N-1)$.

The computing form of the interpolation postprocessing in the bigger subinterval $\tilde{I}_{\frac{n+1}{2}}(n=$ $1,3, \cdots, N-1)$ is as follows.

For $t \in \tilde{I}_{\frac{n+1}{2}}:=\left[t_{n-1}, t_{n+1}\right](n=1,3, \ldots, N-1)$ :

$$
I_{2 h}^{2 m-1} u_{h}(t)=\sum_{j=1}^{2 m} \tilde{u}_{\frac{n+1}{2} j} \tilde{L}_{\frac{n+1}{2} j}(t) .
$$

Where the Lagrange basis functions $\tilde{L}_{\frac{n+1}{2} j}(t)(j=1,2, \ldots, 2 m)$ are constructed by the $2 m$ collocation points. Because of the local property of the basis function, it is very easy to show that:

$$
I_{2 h}^{2 m-1} u_{h}(t)=\sum_{j=1}^{m} u_{n, j} \tilde{L}_{\frac{n+1}{2} j}(t)+\sum_{j=m+1}^{2 m} u_{n+1, j} \tilde{L}_{\frac{n+1}{2} j}(t) .
$$

Therefore, the computing time is mainly composed by the computation of the higher order Lagrange polynomials, which greatly simplifies calculation.

Iteration postprocessing solution $u_{h}^{i t}(t)$

For the iteration postprocessing, we compute the new approximation $u_{h}^{i t}(t)$ at $t=t_{n-1}+$ $v h_{n} \in I_{n}=\left[t_{n-1}, t_{n}\right]$ with $v \in[0,1]:$

$$
\begin{aligned}
u_{h}^{i t}(t)= & g(t)+\int_{0}^{t}(t-s)^{-\alpha} K(t, s) u_{h}(s) d s \\
= & g(t)+\sum_{l=1}^{n-1} \int_{t_{l-1}}^{t_{l}}(t-s)^{-\alpha} K(t, s) u_{h}(s) d s+\int_{t_{n-1}}^{t}(t-s)^{-\alpha} K(t, s) u_{h}(s) d s \\
= & g(t)+\sum_{l=1}^{n-1} \sum_{j=1}^{m} u_{l, j} \int_{t_{l-1}}^{t_{l}}(t-s)^{-\alpha} K(t, s) L_{l, j}(s) d s \\
& +\sum_{j=1}^{m} u_{n, j} \int_{t_{n-1}}^{t}(t-s)^{-\alpha} K(t, s) L_{n, j}(s) d s \\
= & g(t)+\sum_{l=1}^{n-1} \sum_{j=1}^{m} u_{l, j} h_{l} \int_{0}^{1}\left(t-t_{l-1}-s h_{l}\right)^{-\alpha} K\left(t, t_{l-1}+s h_{l}\right) L_{j}(s) d s \\
& +\sum_{j=1}^{m} u_{n, j} h_{n}^{1-\alpha} \int_{0}^{v}(v-s)^{-\alpha} K\left(t, t_{n-1}-s h_{n}\right) L_{j}(s) d s .
\end{aligned}
$$

Because of the weak singularity of the kernel, the normal numerical integral is no longer valid. We must divide the interval $[0, v]$ into several subintervals (using graded mesh) and compute 
numerical integral in each subinterval (omitting the subinterval containing the singular point $v$ ). This increases the complexity of the iteration solution $u_{h}^{i t}(t)$. (See Kaneko and Xu 1994 for details).

\section{Numerical experiments}

In this section, we give an example to illustrate the theory established in the previous section.

Example 4.1 We solve the following second kind weakly singular VIE:

$$
y(t)=f(t)+\int_{0}^{t} \frac{1}{\sqrt{|t-s|}} y(s) d s, \quad t \in[0,1],
$$

where $f(t)$ is given to make the exact solution $y(t)=\sqrt{t}$.

We first compute the piecewise linear $(m=2)$ collocation solution $u_{h}$ under graded mesh, and obtain two different interpolated collocation solutions $I_{2 h}^{2 m-1} u_{h}$ (27) and $I_{2 h}^{m} u_{h}$ (33). The numerical results and error figures for the error estimates are given in Table 1 and Fig. 1.

We see from Table 1 and Fig. 1 that:

$$
\begin{aligned}
& \left\|u-u_{h}\right\|_{\infty}=\mathcal{O}\left(h^{m}\right),\left\|u-I_{2 h}^{2 m-1} u_{h}\right\|_{\infty}=\mathcal{O}\left(h^{m+1-\alpha}\right), \\
& \left\|u_{h}-P_{h} u\right\|_{\infty}=\mathcal{O}\left(h^{m+1-\alpha}\right)\left\|u-I_{2 h}^{m} u_{h}\right\|_{\infty}=\mathcal{O}\left(h^{m+1-\alpha}\right)(m=2) .
\end{aligned}
$$

We calculate the interpolated hybrid collocation solution $\hat{I}_{2 h} u_{h}$ based on the piecewise quadratic hybrid collocation solution $u_{h}(m=3)$. As mentioned in Sect. 3.3, in practical computation, we just need to calculate the interpolated hybrid collocation solution $\tilde{I}_{2 h} u_{h}$ by the least-squares method in the first two subintervals $I_{1} \cup I_{2}$. We also make a comparison between the interpolated hybrid collocation solution and the iterated hybrid collocation solution. The relevant numerical results are illustrated in Table 2.

We see from Table 2 that the convergence order for the interpolated hybrid collocation solution is $\mathcal{O}\left(h^{m+1-\alpha}\right)$ and the CPU time of getting the interpolated hybrid collocation solution is much less than that of getting the iterated hybrid collocation solution which shows the efficiency our proposed method.
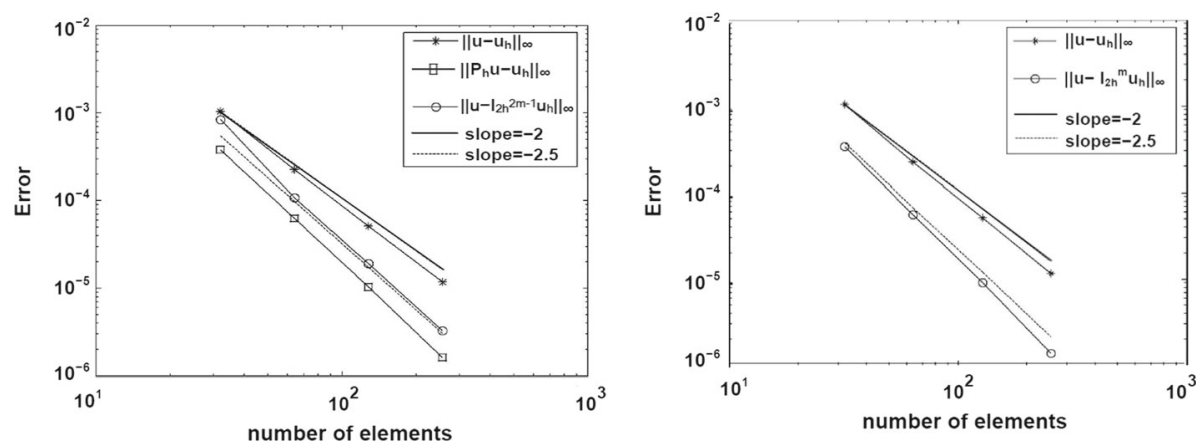

Fig. 1 Superclose and superconvergence (left) and least-squares postprocessing (right) 


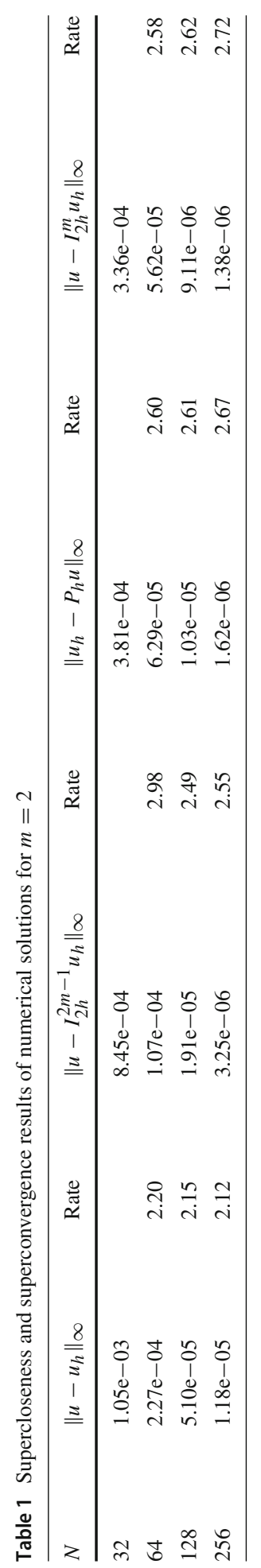




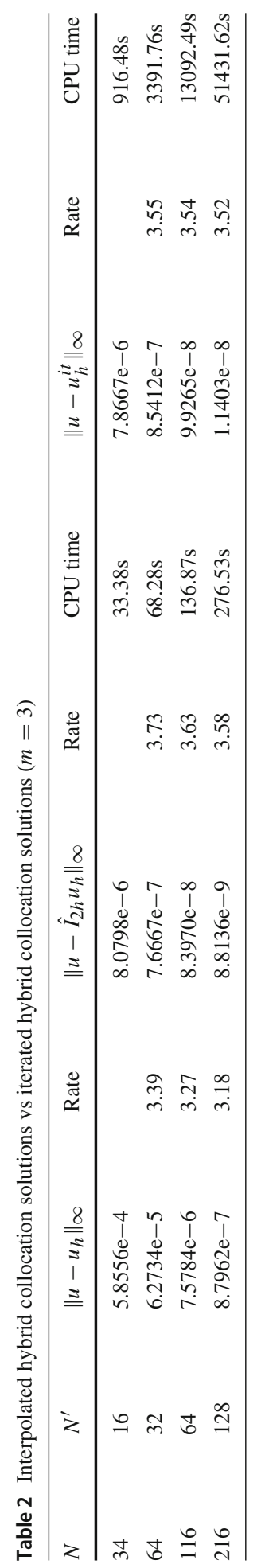

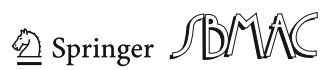




\section{Concluding remarks}

In this paper, we propose two different interpolation postprocessing approximations of higher accuracy based on the collocation solutions and the hybrid collocation solutions. The superconvergence orders of the proposed methods are the same as those of the iterated methods, but are much simpler in computation. The following problem remains to be addressed in future research work:

1. The nonpolynomial spectral methods for high-dimensional IEs.

2. The new numerical method combining the multistep collocation and the hybrid collocation method and aiming to reduce the computational complexity of the numerical solution for IEs.

Acknowledgements This work was supported by National Natural Science Foundation of China (Grant No. 11971047).

Open Access This article is licensed under a Creative Commons Attribution 4.0 International License, which permits use, sharing, adaptation, distribution and reproduction in any medium or format, as long as you give appropriate credit to the original author(s) and the source, provide a link to the Creative Commons licence, and indicate if changes were made. The images or other third party material in this article are included in the article's Creative Commons licence, unless indicated otherwise in a credit line to the material. If material is not included in the article's Creative Commons licence and your intended use is not permitted by statutory regulation or exceeds the permitted use, you will need to obtain permission directly from the copyright holder. To view a copy of this licence, visit http://creativecommons.org/licenses/by/4.0/.

\section{References}

Atkinson K (1997) The numerical solution of integral equations of the second kind. Cambridge University Press, Cambridge, pp 100-156

Brunner H (1983) Nonpolynomial spline collocation for Volterra equations with weakly singular kernels. SIAM J Numer Anal 20:1106-1119

Brunner H (1984) Iterated collocation methods and their discretizations for Volterra integral equations. SIAM J Numer Anal 21:1132-1145

Brunner H (1985) The numerical solution of weakly singular Volterra integral equations by collocation on graded meshes. Math Comp 45:417-437

Brunner H, Yan N (1992) On global superconvergence of iterated collocation solutions to linear second-kind Volterra integral equations. J Comput Math 10:348-357

Brunner H (2004) Collocation methods for Volterra integral and related functional differential equations. Cambridge University Press, Cambridge, pp 53-418

Cao Y, Herdman T, Xu Y (2003) A hybrid collocation method for Volterra integral equations with weakly singular kernels. SIAM J Numer Anal 41:364-381

Diogo T (2009) Collocation and iterated collocation methods for a class of weakly singular Volterra integral equations. J Comput Appl Math 229:363-372

Diogo T, McKee S, Tang T (1994) Collocation methods for second-kind Volterra integral equations with weakly singular kernels. Proc R Soc Edinb 124:199-210

Graham I, Joe S, Sloan IH (1985) Iterated Galerkin versus iterated collocation for integral equations of the second kind. IMA J Numer Anal 5:355-369

Hu Q (1996) Stieltjes derivatives and $\beta$-polynomial spline collocation for Volterra integro-differential equations with singularities, SIAM. J Numer Anal 33:208-220

Hu Q (1997) Superconvergence of numerical solutions to Volterra integral equations with singularities. SIAM J Numer Anal 34:1698-1707

Huang Q, Zhang S (2010) Superconvergence of interpolated collocation solutions for Hammerstein equations. Numer Methods Partial Differ Equ 26:290-304

Huang Q, Xie H (2009) Superconvergence of the interpolated Galerkin solutions for Hammerstein equations. Int J Numer Anal Model 6:696-710 
Huang M, Xu Y (2006) Superconvergence of the iterated hybrid collocation method for weakly singular Volterra integral equations. J Integral Equ Appl 18:83-116

Kaneko H, Xu Y (1994) Gaussian-type quadratures for weakly singular integrals and their applications to the Fredholm integral equation of the second kind. Math Comput 62:739-753

Lin Q, Lin J (2006) Finite element methods: accuracy and improvement. Science Press, Beijing

Lin Q, Yan N, (1996) The construction and analysis of high efficiency finite element methods. Hebei University Publishers (in Chinese)

Lin Q, Zhang S, Yan N (1998) An acceleration method for integral equations by using interpolation postprocessing. Adv Comp Math 9:117-129

Naga A, Zhang Z (2004) A posteriori error estimates based on the polynomial preserving recovery. SIAM J Numer Anal 42:1780-1800

Pedas A, Vainikko G (2004a) Numerical solution of weakly singular Volterra integral equations with change of variables. Proc Eston Acad Sci Phys Math 53:96-106

Pedas A, Vainikko G (2004b) Smoothing transformation and piecewise polynomial collocation for weakly singular Volterra integral equations. Computing 73:271-293

Rebelo M, Diogo T (2010) A hybrid collocation method for a nonlinear Volterra integral equation with weakly singular kernel. J Comput Appl Math 234:2859-2869

Sloan IH (1976) Improvement by iteration for compact operator equations. Math Comput 30:758-764

Sloan IH (1990) Superconvergence. In: Golberg M (ed) Numerical solution of integral equations. Plenum, New York, pp 35-70

Tang T (1992) Superconvergence of numerical solutions to weakly singular Volterra Integral differential equations. Numer Math 61:373-382

Zhang S, Lin Y, Rao M (2000) Numerical solutions for second-kind Volterra integral equations by Galerkin methods. Appl Math 45:19-39

Zhang Z, Naga A (2005) A new finite element gradient recovery method: superconvergence property. SIAM J Sci Comput 26:1192-1213

Publisher's Note Springer Nature remains neutral with regard to jurisdictional claims in published maps and institutional affiliations. 